Questions vives

\section{Questions Vives}

Recherches en éducation

$N^{\circ} 30 \mid 2018$

Les effets formatifs du mémoire en formation initiale des enseignants

\title{
Mémoire et travail scientifique de nature réflexive dans la formation initiale des enseignants - regards croisés sur ces objets
}

Memory and scientific work of a reflective nature in the initial training of teachers cross-referencing these objects

\section{Sylvie Dozolme}

\section{(2) OpenEdition}

\section{Journals}

\section{Édition électronique}

URL : http://journals.openedition.org/questionsvives/3449

DOI : $10.4000 /$ questionsvives.3449

ISSN : 1775-433X

\section{Éditeur}

Université Aix-Marseille (AMU)

\section{Édition imprimée}

Date de publication : 21 décembre 2018

ISBN : 978-2-912643-54-4

ISSN : 1635-4079

\section{Référence électronique}

Sylvie Dozolme, "Mémoire et travail scientifique de nature réflexive dans la formation initiale des enseignants - regards croisés sur ces objets », Questions Vives [En ligne], № 30 | 2018, mis en ligne le 12 décembre 2018, consulté le 01 mai 2019. URL : http://journals.openedition.org/ questionsvives/3449; DOI : 10.4000/questionsvives.3449

Ce document a été généré automatiquement le 1 mai 2019.

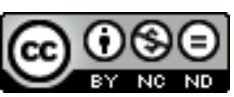

Questions Vives est mis à disposition selon les termes de la licence Creative Commons Attribution -

Pas d'Utilisation Commerciale - Pas de Modification 4.0 International. 


\title{
Mémoire et travail scientifique de nature réflexive dans la formation initiale des enseignants - regards croisés sur ces objets
}

\author{
Memory and scientific work of a reflective nature in the initial training of \\ teachers cross-referencing these objects
}

Sylvie Dozolme

\section{L'écrit réflexif, une exigence institutionnelle}

1 En juillet 2013, avec la Loi de refondation de l'école, la formation des enseignants et personnels d'éducation s'est dotée des Écoles supérieures du professorat et de l'éducation (ESPE), composantes des universités auxquelles elles sont rattachées. Cette loi crée également un comité national ad hoc, chargé du suivi de cette réforme. Concurremment, l'arrêt no 0200 du 29 août 2013 fixe le cadre du master Métiers de l'enseignement, de l'éducation et de la formation (MEEF). Ainsi sont posées ces deux bases.

\subsection{L'entrée dans le métier d'enseignant en France depuis 2013}

2 Titularisés à un niveau master, les nouveaux enseignants se retrouvent dans des obligations de diplomation similaires à celles de leurs collègues européens. La France se situe dans la mouvance européenne puisque, dans vingt-quatre pays de l'Union européenne, le nombre d'années de formation initiale requises se situe entre quatre et cinq ans (Lefresne \& Fournier, 2016). Le législateur a fixé un cadre national des formations conduisant à la délivrance des diplômes nationaux, notamment ceux de master dans son arrêté du 22 janvier 2014. À l'article 16, ce texte précise que «la formation comprend obligatoirement une initiation à la recherche et, notamment, la 
rédaction d'un mémoire ou d'autres travaux d'études personnels ». Le master MEEF ne déroge pas à ce texte puisqu'il stipule également dans son article 19 :

Dans le cadre du stage de la formation en alternance du master « MEEF », chaque étudiant réalise un mémoire de master qui doit avoir un contenu disciplinaire et de recherche en relation avec la finalité pédagogique et les pratiques professionnelles. Le mémoire prend appui sur le stage de la formation en alternance et sur d'autres enseignements au sein de la formation.

3 Cependant, moins d'un an après la création du master MEEF ${ }^{1}$, le recteur Filâtre (2015), président du comité national de suivi, tire une première sonnette d'alarme sur le mémoire avec des interrogations tant sur les conditions de sa réalisation que sur son contenu. Face aux hétérogénéités observées, une note en date du 28 octobre 2015, émanant du ministère de l'Éducation nationale, de l'Enseignement supérieur et de la Recherche, est envoyée aux directeurs des EPSE. Elle vient préciser les modalités de mise en œuvre du mémoire dans le cadre de la formation aux métiers de l'enseignement, de l'éducation et de la formation. Forte de deux années d'expérience, cette note affiche clairement ses ambitions :

Préciser les contours et les attendus du mémoire de master MEEF, mais aussi d'apporter aux équipes en charge de la formation des enseignants et des personnels d'éducation des préconisations sur la place et la nature d'un travail scientifique de nature réflexive dans la formation des fonctionnaires bénéficiaires d'un parcours de formation adapté.

Outre la réitération des mêmes constats d'hétérogénéité évoqués par Filâtre (2015), cette note définit clairement les natures des écrits attendus selon les situations pour le moins multiples dans lesquelles les étudiants fonctionnaires stagiaires (EFS) peuvent se trouver. Ainsi, elle institutionnalise le travail scientifique réflexif (TSR) attendu par les EFS non inscrits en master MEEF $2^{\mathrm{e}}$ année. Elle précise également que les exigences attendues sont de même nature pour le mémoire que pour le TSR. Cependant, les modalités doivent être adaptées à la diversité des étudiants fonctionnaires stagiaires accueillis par l'ESPE.

Tableau 1 : diversité des situations des EFS

\begin{tabular}{|l|l|}
\hline Situation de l'EFS lors de son entrée en stage & Écrit attendu \\
\hline EFS déjà titulaire d'un Master MEEF & $\begin{array}{l}\text { TSR pouvant se situer dans le prolongement } \\
\text { des travaux conduits dans le cadre de son } \\
\text { mémoire MEEF }\end{array}$ \\
\hline EFS titulaire d'un titre Bac + 5 hors MEEF & $\begin{array}{l}\text { Mémoire ou TSR pouvant être le prolongement } \\
\text { de travaux précédent, mais avec une visée } \\
\text { professionnelle enseignante }\end{array}$ \\
\hline EFS dispensé de titre & Adaptation au cas par cas \\
\hline $\begin{array}{l}\text { EFS dispensé de titre ou détenant un master } \\
\text { hors MEEF, mais justifiant d'une expérience } \\
\text { significative en enseignement }\end{array}$ & $\begin{array}{l}\text { Affecté à temps plein en établissement } \\
\text { collectif de recherche qui l'intéresse }\end{array}$ \\
\hline
\end{tabular}

5 De cette note, trois éléments saillants méritent notre attention. Le premier point concerne la forte recommandation de commencer le travail de recherche que doivent 
conduire les étudiants dès la première année. Or, plusieurs cas de figure coexistent parmi les EFS : 1) tous les étudiants en M1 MEEF ne réussissent pas le concours, d'où des départs de formation et des changements de projet professionnel pour les non-lauréats ; 2) tous les étudiants de M1 MEEF lauréats de concours du second degré ne restent pas dans l'ESPE où ils ont étudié en $1^{\text {re }}$ année ; 3 ) la confrontation au terrain dans le cadre de la formation par alternance en M2 peut les conduire à changer de thème ou de problématique ;4) la présence conséquente en M2 MEEF d'étudiants non issus d'un M1 MEEF donc qui n'ont pas eu les cours de M1 et la préorientation vers des thématiques.

6 Deuxième point qui interroge : cette note précise que le mémoire, du fait de l'alternance de la formation, ne doit pas être envisagé par les EFS « comme un exercice constituant une charge supplémentaire ». Face à ces mots, des interrogations surgissent : comment est-ce possible? Comment une réflexion peut-elle se faire sans prendre du temps sur d'autres activités? Comment des documents scientifiques peuvent-ils être lus sans déborder sur l'emploi du temps serré des EFS ?

7 Enfin, dernier point saillant que nous avons relevé dans les préconisations de cette note : «le mémoire est mené sous la responsabilité d'un enseignant-chercheur». L'encadrement et le suivi d'un mémoire ou d'un TSR sont valorisés à concurrence de 3 HTD (quotité moyenne pour cette activité). Pour une ESPE comportant $400 \mathrm{EFS}^{2}$, cela revient à employer à temps plein 6,25 enseignants-chercheurs, sans autres missions et enseignements possibles. Où trouver ces enseignants-chercheurs (EC) quand les chiffres remontés au Réseau national par les ESPE mentionnent un taux moyen d'EC dans ces écoles de $30 \%$ des enseignants (Ginestié, 2017)?

\subsection{Une injonction forte d'adosser la formation initiale des enseignants à la recherche}

8 Tout en explicitant l'objectif d'une démarche qui se veut scientifique, le recteur Filâtre (2017) revient sur la genèse des ESPE et sur l'adossement à la recherche des formations des enseignants: «en plaçant chaque ESPE au sein de l'écosystème universitaire de l'académie, le législateur a voulu conforter la place de la recherche dans l'activité professionnelle des personnels de l'enseignement et d'éducation et enrichir l'évolution de leur métier » (p. 63). Cette ambition ne se limite pas à la formation initiale, mais devrait irriguer la formation tout au long de la vie des professionnels de l'enseignement, de l'encadrement et de la formation. Des financements « projet investissement avenir » de la vague 2 (PIA 2) ont été dédiés à une politique volontariste souhaitant allier formation, recherche et terrain autour de l'éducation. Cette multiplicité des travaux amène au constat, non pas d'une recherche en sciences de l'éducation, mais de plusieurs types de recherches en sciences en éducation, tant les sphères sont nombreuses (Ginestié, 2017). La diversité des recherches conduites en sciences de l'éducation devrait offrir aux enseignants, et en l'occurrence aux débutants, des thématiques à explorer en phase avec leurs préoccupations professionnelles.

Les écrits produits durant l'année de stagiaire d'enseignants débutants semblent devoir occuper une place prépondérante dans la formation puisqu'ils sont jugés comme « la clef de voûte de la formation » (Filâtre, 2017, p. 63). Dans sa lettre de rentrée adressée aux enseignants, en date du 6 septembre 2017, le président de la République française réitère cette démarche de recherche scientifique dans la formation initiale des enseignants : « La 
formation des futurs professeurs doit être davantage nourrie par les recherches scientifiques, les expérimentations et la comparaison internationale ».

Ce travail représente un investissement majeur pour les stagiaires (Campion, 2017), mais les contraintes temporelles qu'ils connaissent empêcheraient de fournir un travail complètement abouti (Bruillard, 2017). Si un équilibre entre les bénéfices et les contraintes professionnelles serait donc à trouver, une synthèse de certains travaux produits dans ces domaines permettrait une meilleure préhension des enjeux.

\section{L'écriture scientifique au bénéfice de la professionnalisation}

11 Déjà désacralisée à la fin des années 1960 dans les années 1968 (Bouquet, 2009), l'écriture a pris un rôle central dans les formations universitaires, accru en cela par la mise en place du système LMD. Ces écrits professionnels et/ou scientifiques revêtent différentes formes, que ce soit des rapports, des études, des comptes rendus, des mémoires, des travaux réflexifs et autres travaux d'écriture. Ces activités langagières, quelle que soit leur forme, tendraient à faciliter l'acquisition d'une profession et, en l'occurrence, celle des professionnels de l'éducation et de la formation. C'est ce qui a conduit les chercheurs à s'intéresser aux liens entre écriture et professionnalisation, en interrogeant les apports de l'écriture dans des dispositifs de formation, de recherche, d'accompagnement de pratiques et en évaluant leur caractère scientifique.

\subsection{La notion de profession et mots dérivés}

De fortes attentes émanent donc de l'institution afin de trouver des modalités autour de l'entrée dans le métier des enseignants et tendant à la favoriser. Les notions de professionnalité et professionnalisation sont utilisées, sciemment ou non, dans diverses recommandations et injonctions pour la formation des enseignants débutants.

Pour une définition de la racine étymologique commune de ces mots, nous nous référons à la sociologie des professions. Dubar, Tripier et Boussard (2011) distinguent quatre sens au mot profession, dont l'origine latine professio renvoie à un premier qui est de déclarer sa foi (profession de foi). En son deuxième sens, la profession correspond à l'emploi occupé qui permet de subvenir à ses besoins. Le troisième sens correspond aux personnes nommées par un même nom de métier, réalisant une activité semblable. Enfin, la profession est aussi "une fonction, une compétence reconnue au sein d'une organisation » (p. 12). La notion de reconnaissance serait au cœur de la signification du mot, actualisé par le discours politique. Être un professionnel impliquerait le désir d'une (re)connaissance de son métier par son entourage, et par là même, le souhait que sa vision modifie l'image qu'il en a. Cela conduirait le professionnel à modifier lui-même la représentation qu'il aurait de son métier.

Hughes (1996) rejoint les deux notions de profession et de professionnalisation par cette modification collective du symbole véhiculé par le métier :

Le terme de profession est un symbole de la conception du travail qui est revendiquée, et par suite un symbole de moi. La tendance à la «professionnalisation » d'un métier correspond donc à la mobilité collective d'une partie de ses membres (p. 77). 
Dans ses travaux, Wittorski (2008a, p. 32) considère que la professionnalisation renvoie à trois notions. Premièrement, "une intention» de la part de l'organisation avec une «mise en mouvement» des individus, cette mise en mouvement se traduisant par la proposition d'une offre de formation. Deuxièmement, « un processus » d'action dont les instigateurs seraient les individus ou les groupes dans lesquels ils sont constitués ; de leur part émanerait une demande de reconnaissance de leur action par l'organisation. Troisièmement, "une transaction" qui aurait comme objectif "l'attribution d'une professionnalité à l'individu à partir des process d'action développés». Selon les pays, la professionnalisation revêt des nuances. Dans les pays anglo-saxons, elle est davantage considérée comme un processus qui va transformer une activité en profession. Alors que pour Wittorski (2008a) comme pour Bourdoncle (2000), sa finalité serait davantage dans la reconnaissance sociale obtenue par les savoirs professionnels, idée précédemment évoquée par Lang (1999). Ce dernier rajoute une double notion de développement de compétences et de nouveau métier face aux évolutions. Associée à ce développement professionnel, une construction identitaire se mettrait également en place :

Dans un contexte de professionnalisation, [...] le dispositif proposé à l'individu constitue une offre identitaire (c'est-à-dire une « injonction » à devenir conforme à des attentes exprimées par l'organisation) pouvant entrer en congruence ou en conflit avec le projet que poursuit le sujet pour lui-même (Wittorski, 2008b, pp. 27-28).

16 Appliquée à l'entrée dans le métier des jeunes enseignants, la commande du travail écrit s'inscrirait dans une démarche de professionnalisation. Cependant, sont liées à cet impact positif ou négatif les attentes des EFS face à cet objet. L'impact peut se trouver amplifié selon la place qu'accordent les individus en formation à leur dynamique de changement intérieur (Barbier, 1996).

La professionnalisation recoupe donc les notions de formation, d'identité et de professionnalisation, éléments prépondérants dans notre étude. De plus, cette professionnalisation requiert une forme adhésion de la part des étudiants. L'écrit peut concourir à cela.

\subsection{Le rôle de l'écriture dans la professionnalisation}

L'écriture est un support privilégié tant dans sa dimension formative qu'évaluative. Ainsi, dans leurs travaux, Morisse, Lafortune et Cros (2012) déterminent son impact et le résument :

L'écriture conduit [...] les personnes apprenantes à s'interroger sur le sens de ce qu'elles font, de ce qu'elles apprennent, à articuler leur réflexion sur des expériences personnelles ou professionnelles, à se situer ou se positionner dans les discours théoriques de façon argumentée et à exercer une réflexion critique (p. 2).

L'acte d'écriture aide à la structuration de ses idées, permet d'introduire de la rigueur dans la perception des choses, d'organiser cognitivement l'expérience vécue (Bouissou \& Aroq, 2005 ; Lahire, 1998). Il oblige également l'auteur à prendre un rapport distancié, position qualifiée d'" exotopie ", tout en explicitant sa pensée. Cependant, écrire est aussi un acte chronophage qui oblige à mettre en forme les savoirs expérientiels, ceux qui sont issus de lectures, à donner une existence matérielle aux pensées. Cet acte d'écriture ne peut laisser son acteur neutre. Il s'agit d'une écriture performative (Morisse et al., 2012) qui est produite afin d'expliciter, d'argumenter, d'avoir un effet sur le lecteur. Cet effet se retourne vers son auteur, impactant son agir et sa pensée immédiats et à terme (Crinon \& 
Guigue, 2006). L'écriture provoque donc des «retours réflexifs » (Schön, 1994) sur l'action, point développé ci-après.

Toutefois, cette vision de l'écriture est nuancée (Chartier, 2003 ; Crinon \& Guigue, 2006 ; Reuter, 2004). Malgré les points positifs énoncés, il s'agit d'un travail ardu qui est doublement conditionné, par le rapport à l'écriture que les étudiants ont et par l'engagement qu'ils mettent en œuvre. Si l'écrit est consubstantiel à des activités sociales spécifiques et à un rapport particulier à l'écriture, il implique l'acceptation par le scripteur de mettre en cohérence ce qu'il écrit avec ce qu'il réalise. Ce point convoque la théorie de l'engagement telle que Beauvoir et Deschamps (1990, p. 35) la présentent : « un acte en tant que tel, peut être générateur d'activité et de modifications cognitives, voire de comportements nouveaux ». Le retrait des étudiants à l'égard de ce travail peut être perçu comme un refus de s'engager dans un travail pour lequel la cohérence entre la formation et le terrain demeure inexistante (Crinon \& Guigue, 2006) ou pour lequel le statut de cet écrit est abscons. Les attendus universitaires et institutionnels, les relations professionnelles et directoriales, la circulation du document finalisé interrogent les étudiants sur ce qu'il convient d'écrire et les risques qu'ils peuvent encourir au terme de la circulation de leur document. S'ensuit une réserve dans l'écriture qui donne un contenu aseptisé, dans un langage prudent, limitant voire éliminant l'intérêt formatif (Chartier, 2003). Autre aspect sur lequel les étudiants s'interrogent : l'aspect scientifique exprimé dans les textes de cadrage de la formation.

\subsection{La notion de scientificité}

Déterminer l'aspect scientifique d'un écrit semble relever de la gageure. Berthelot (2003) identifie trois traits servant à le définir : un objectif de produire de la connaissance, la reconnaissance par une communauté de l'atteinte de cet objectif et l'intention de publication. Mroue (2014), dans sa thèse, explore les contours de ce type d'écrit sans pour autant définir stricto sensu la notion. De ses recherches ressortent les notions de spécialité, de terminologie, d'originalité, de communication, d'autonomie, de pratiques sociales et communautaires... un écrit qu'elle qualifie de " polyphonique ». Est également évoquée la nécessité d'une acculturation à l'écrit de recherche, procurant des tensions durant l'appropriation de ce processus par les étudiants. Les tensions soulignées proviendraient autant des contraintes imposées par la structuration de la pensée que celles qui sont liées aux normes d'écriture. Dans les contraintes de fond, cet écrit présente la particularité d'être à la fois validé par la communauté discursive et empreint de la diversité des discours d'autrui traversant ceux des étudiants. Pour les contraintes liées à la forme, les différentes compétences à mettre en œuvre ${ }^{3}$ se résument par le "savoirécrire ", c'est-à-dire la capacité des étudiants à « graphier » (Dabène, 1993).

L'injonction ministérielle, mentionnée précédemment, précise les attentes autour de ces écrits en utilisant les termes de « recherche » et de «scientifique». Cependant, avec les contraintes professionnelles fortes que connaissent ces EFS, il semble difficile de pouvoir concilier ces différents paramètres et aboutir à un écrit scientifique tel qu'attendu par la communauté scientifique. La distinction faite par Glorieux (2016) sur l'écrit scientifique nuancerait les attendus et justifierait le caractère scientifique évoqué. Elle évoque deux types d'écrit : un «écrit de recherche ou [...] un écrit de recherche en formation » (p. 94). Les attentes institutionnelles rejoindraient davantage la seconde nature, laissant ainsi une place prépondérante à l'analyse de l'activité des EFS, sur eux, par eux. 


\subsection{La place de la réciprocité réflexive}

compétence se construit [...] dans l'interaction entre les personnes et les contextes, selon un double processus d'extériorisation et d'internalisation. [...] Le mémoire [...] stimule le développement de la pensée par ce double mouvement" (Bouissou \& Aroq, 2005, p. 17). La réalisation du mémoire/TSR nécessite que l'étudiant entre en contact avec son entourage professionnel. Ainsi, il se construit une posture de réflexivité qui va l'inciter à questionner sa propre pratique (Altet, Bru \& BlanchardLaville, 2012) et à opérer de la distanciation à son égard (Bouissou \& Aroq, 2005). Cette finalité du travail est donc d'ordre praxéologique, offrant à l'étudiant des pistes de réflexion et/ou d'action. À côté de cette fonction praxéologique se trouve une fonction heuristique, c'est-à-dire qui favorise l'intégration de savoirs différents, permettant l'émergence d'une culture professionnelle réfléchie.

Cette posture de réflexivité, construite au cours de l'année de stage, renvoie aux travaux de Schön (1994, p. 97) :

[Le professionnel] ouvre la porte à des situations qu'il trouve incertaines ou particulières où il se met en état de surprise, de perplexité et de confusion; il réfléchit aux phénomènes qu'il constate et aux premières conclusions que son comportement lui a implicitement dictées; ensuite, la poursuite de l'expérimentation lui permet de comprendre les phénomènes autrement, et de transformer la situation (p. 97).

Si une réflexion sur sa propre activité, en réponse à des échanges entre pairs, à des observations sur le terrain, demeure indéniable, la professionnalisation des nouveaux enseignants ne saurait se limiter à cela. Des écrits notent la faiblesse et le risque que générerait une formation basant la professionnalisation principalement sur cette activité, en lien avec l'écrit demandé. Dans les travaux de la sociologie dispositionnelle, des interrogations pointent le fait que les compétences acquises par le biais de la réflexivité ne seraient pas acquises ad vitam ceternam et pas nécessairement transposées ou transposables. Par ailleurs, il existe une forte variabilité interindividuelle des positionnements des étudiants face aux travaux écrits et également une réelle variabilité intra-individuelle (Bouissou \& Aroq, 2005), l'homme n'étant «ni une construction logique, ni exempt de contradictions psychologiques» (Lahire, 2001, p. 146).

Sous certaines conditions, l'écriture comme la posture réflexive permettent aux étudiants de poser des bases pour leur professionnalisation. Les spécificités du métier d'enseignant et de son entrée en fonction peuvent modérer ces avantages. Afin d'appréhender cela, notre étude cherche à interroger des EFS du second degré, du personnel d'éducation et leurs formateurs autour de ces écrits. Ces travaux conduits dans une ESPE de taille moyenne ${ }^{4}$ visent à aborder trois axes : la définition même de ces objets (mémoire et TSR) par les acteurs, l'encadrement de ce travail, de son écriture à sa soutenance, ainsi que son impact dans la professionnalisation de ces débutants.

\section{Méthodologie}

Un questionnaire de 41 items a été adressé en juin 2017 aux étudiants de master MEEF de l'ESPE Clermont-Auvergne, mais également aux étudiants des DU «enseigner dans le second degré5 $»$, DU «approfondissement pour enseigner dans le second degré ${ }^{»}$, DU 
« encadrement éducatif » et DU «approfondissement pour l'encadrement éducatif ». Ce questionnaire visait à interroger les EFS sur la manière dont ils appréhendaient le mémoire. Dans le cadre de cette étude, nous nous sommes focalisée sur les 230 EFS du second degré et de l'encadrement éducatif. Ont donc été exclus de fait les étudiants de M2 MEEF non fonctionnaires stagiaires.

\subsection{Méthodologie mixte mise en œuvre}

Le questionnaire, administré par le site Sondageonline, était composé de sept parties :

- identification du répondant : âge, genre, diplôme dans lequel il est inscrit, dernier diplôme obtenu, réalisation dans le cursus universitaire antérieur d'un mémoire ou d'un travail réflexif ;

- formation à la méthodologie : nombre d'heures de cours, appréciation sur la quantité de ces heures, remarques sur cet enseignement ;

- le mémoire ou le travail réflexif : définition de l'objet ; évaluation des difficultés rencontrées pour trouver le thème, le sujet, la bibliographie, mettre en place une collecte de données, faire le lien entre les cours et le document à produire ; le moment de l'année universitaire où l'étudiant a commencé son travail d'écriture ;

- encadrement du travail: évaluation des difficultés pour comprendre la mission d'un directeur de mémoire/travail réflexif, trouver les modalités d'échange avec lui, le rencontrer ;

- écriture du travail : évaluation des difficultés pour comprendre les attendus, rédiger le travail demandé, organiser les différentes parties, formaliser les citations ; place et rôle du directeur de mémoire/travail réflexif durant la phase d'écriture ;

- soutenance du mémoire (le travail réflexif ne donnant pas lieu à soutenance dans cette ESPE) : personnes présentes, conseils sollicités et reçus pour cette épreuve, le ressenti face à cette épreuve, l'attitude perçue du jury ;

- bilan et projection de ce travail : éléments que l'étudiant aurait changés dans son travail, impact de ce travail dans son métier (à moyen et long terme), projet de faire une thèse en sciences de l'éducation.

29 Adressé au terme de l'année universitaire 2016-2017, il visait à recueillir le ressenti des étudiants lorsque le travail demandé a été réalisé et la soutenance passée. De plus, cette période de l'année nous a semblé offrir plus de disponibilité pour leur permettre de répondre. Nonobstant, elle correspondait également à une étape charnière dans leur début professionnel, composée de la mutation et de la titularisation. Préoccupés par l'organisation de leur nouvelle installation professionnelle, ils avaient d'autres préoccupations que de remplir un questionnaire en ligne.

Un second questionnaire a été adressé aux quarante-trois formateurs identifiés comme étant soit enseignants dans les cours de méthodologie de recherche soit encadrants de mémoires ou de travaux réflexifs, ou les deux. Il a été administré selon les mêmes modalités pendant la même période. Composé de questions similaires, il était structuré également en sept parties :

- identification du répondant: âge, genre, statut au sein de l'ESPE, nombre d'années dans l'activité de cours/accompagnement d'écrits, activités similaires dans une autre composante de l'université ;

- cours de méthodologie de recherche: activité d'enseignement, nombre d'heures réalisées par année universitaire, jugement sur le nombre d'heures ; 
- encadrement du travail : évaluation des difficultés que peuvent ressentir les étudiants dans les différentes phases de, la conception jusqu'au positionnement attendu pour la soutenance ;

- professionnalisation des étudiants par ce travail d'écriture : définition de l'objet, pertinence à le considérer comme un objet « en soi » indépendamment de la formation ;

- temporalité et impact du mémoire: évaluation sur le positionnement, les objectifs et l'impact de ce travail dans la professionnalisation des enseignants stagiaires ;

- soutenance du mémoire : rôle joué par le formateur, le sentiment que les EFS peuvent ressentir face à cet oral ;

- retour sur les anciennes promotions: contacts avec elles, nature des échanges, impact professionnel de ces écrits sur la professionnalisation.

31 Ce questionnaire souhaitait recueillir le ressenti des formateurs autour de ces objets communs d'écriture. Il permettait également de mettre en tension les données collectées des deux familles de répondants, tout en étant contigu à la soutenance. En dernière question était proposée aux répondants la possibilité de participer à un entretien semidirectif pour revenir sur certaines notions et/ou compléter des réponses pouvant avoir été trop succinctes. Si la fin des cours pouvait offrir la possibilité pour les formateurs de répondre à cette demande, leur participation en tant que jury de concours ou de diplômes ainsi que leur contribution à des colloques ou des séminaires risquait de limiter le nombre de répondants. Nous travaillons donc sur des pratiques déclarées, un discours rapporté et non sur l'observation directe d'échanges entre directeurs et étudiants.

\subsection{Portrait des répondants étudiants}

Sur l'ensemble des étudiants contactés par leur adresse mail universitaire, 64 ont répondu, offrant un taux de réponse de $27,83 \%$. Les tableaux ci-après reprennent les informations collectées sur quelques critères sociodémographiques susceptibles d'apporter un éclairage sur les répondants.

Tableau 2 : diplômes dans lesquels sont inscrits les répondants EFS

\begin{tabular}{|l|l|l|l|l|}
\hline Formations suivies en 2016-2017 & Femmes & Hommes & Total & $\%$ \\
\hline Master MEEF 2d degré & 28 & 20 & 48 & 75 \\
\hline Master MEEF encadrement éducatif & & 1 & 1 & 1,5 \\
\hline DU enseigner dans le 2 degré & 7 & 7 & 14 & 22 \\
\hline DU approfondissement 2 degré & 1 & & 1 & 1,5 \\
\hline Total & 36 & 28 & 64 & 100 \\
\hline
\end{tabular}

Tableau 3 : âge des répondants EFS

\begin{tabular}{|l|l|l|l|l|}
\hline Âge & Femmes & Hommes & Total & $\%$ \\
\hline
\end{tabular}




\begin{tabular}{|l|l|l|l|l|}
\hline Moins de 26 ans & 17 & 16 & 33 & 52 \\
\hline Entre 26 et 30 ans & 6 & 4 & 10 & 16 \\
\hline Entre 31 et 35 ans & 5 & 4 & 9 & 14 \\
\hline Entre 36 et 40 ans & 6 & 1 & 7 & 11 \\
\hline 40 ans et plus & 1 & 3 & 4 & 6 \\
\hline Pas de réponse & 1 & & 1 & 1 \\
\hline Total & 36 & 28 & 64 & 100 \\
\hline
\end{tabular}

Tableau 4 : dernier diplôme obtenu par les répondants EFS

\begin{tabular}{|l|l|l|l|l|}
\hline $\begin{array}{l}\text { Dernier diplôme universitaire obtenu } \\
\text { Formation suivie }\end{array}$ & Femmes & Hommes & Total & $\%$ \\
\hline Licence & 4 & 3 & 7 & 11 \\
\hline Master MEEF 1 ${ }^{\text {re année }}$ & 20 & 14 & 34 & 53 \\
\hline Master MEEF 2 année & & 1 & 1 & 1,5 \\
\hline Bac + 4 autre que MEEF & 2 & 2 & 4 & 6 \\
\hline Bac + 5 autre que MEEF & 9 & 6 & 15 & 24 \\
\hline Diplôme d'ingénieur & 1 & 1 & 2 & 3 \\
\hline Doctorat & & 1 & 1 & 1,5 \\
\hline Total & 36 & 28 & 64 & 100 \\
\hline
\end{tabular}

33 Presque la moitié ( $48 \%$ ) des répondants n'ont pas eu l'occasion dans leur parcours universitaire de réaliser un mémoire ou un TSR. Un quart de ces étudiants avaient écrit un mémoire recherche et $11 \%$ un mémoire professionnel, le même pourcentage un travail réflexif.

\subsection{Portrait des répondants formateurs}

18 formateurs sur 43 recensés ont répondu, donnant ainsi un taux de réponse de $41,86 \%$.

Tableau 5 : âge des répondants formateurs

\begin{tabular}{|l|l|l|l|l|}
\hline Âge & Femmes & Hommes & Total & $\%$ \\
\hline
\end{tabular}




\begin{tabular}{|l|l|l|l|l|}
\hline Moins de 30 ans & 1 & & 1 & 6 \\
\hline Entre 36 et 40 ans & 2 & 2 & 4 & 22 \\
\hline Entre 41 et 45 ans & & 1 & 1 & 6 \\
\hline Entre 46 et 50 ans & 2 & 3 & 5 & 28 \\
\hline Entre 51 et 55 ans & 3 & & 3 & 17 \\
\hline Entre 56 et 60 ans & 3 & & 3 & 17 \\
\hline Plus de 60 ans & 1 & & 1 & 6 \\
\hline Total & 12 & 6 & 18 & 100 \\
\hline
\end{tabular}

Tableau 6 : statut des répondants formateurs à l'ESPE Clermont-Auvergne

\begin{tabular}{|l|l|l|l|l|}
\hline Statut à l'ESPE & Femmes & Hommes & Total & $\%$ \\
\hline PRCE & 5 & 2 & 7 & 39 \\
\hline PRAG & 3 & 2 & 5 & 28 \\
\hline PFA & 1 & & 1 & 6 \\
\hline MCF & 3 & 1 & 4 & 22 \\
\hline PU & & 1 & 1 & 6 \\
\hline Total & 12 & 6 & 18 & 100 \\
\hline
\end{tabular}

Sept formateurs ont répondu positivement à l'invitation consistant à poursuivre l'échange lors d'un entretien. Dans le cadre d'un entretien d'explicitation individuel (Vermersch, 1996), ils ont été invités à revenir sur les points saillants du questionnaire et à les développer. Cela a permis quatre heures d'enregistrement, entièrement retranscrites. Ces entretiens ont donné lieu à une analyse de contenu afin de mettre en tension le ressenti exprimé des formateurs avec celui des EFS.

Tableau 7 : présentation des formateurs interviewés

\begin{tabular}{|l|l|l|l|l|l|}
\hline Code & Sexe & Corps & $\begin{array}{l}\text { Ancienneté } \\
\text { accompagnement }\end{array}$ & $\begin{array}{l}\text { Cours } \\
\text { méthodologie }\end{array}$ & $\begin{array}{l}\text { Nombre mémoires- } \\
\text { TSR suivis par an }\end{array}$ \\
\hline F1 & Féminin & Certifié & 1 an & Non & 4 \\
\hline F2 & Masculin & Certifié & Entre 5 et 10 ans & Oui & 6 à 8 \\
\hline F3 & Féminin & Certifié & Entre 5 et 10 ans & Non & 3 mémoires et 2 TSR \\
\hline
\end{tabular}




\begin{tabular}{|l|l|l|l|l|l|}
\hline F4 & Féminin & Certifié & 1 an & Non & 8 \\
\hline F5 & Féminin & $\begin{array}{l}\text { EC } \\
\text { MCF }\end{array}$ & 10 ans & Oui & Entre 25 et 30 \\
\hline F6 & Masculin & Agrégé & Entre 5 et 10 ans & Non & $6-7$ \\
\hline F7 & Masculin & $\begin{array}{l}\text { EC } \\
\text { MCF }\end{array}$ & 10 ans & Oui & 3 mémoires et 8 TSR \\
\hline
\end{tabular}

\subsection{Analyse des données}

Le logiciel en ligne Sondageonline offre diverses fonctionnalités qui facilitent le dépouillement des réponses. Par le biais de requêtes, les réponses sont regroupées selon les critères renseignés par les questionnés. Pour les questions libres ou celles proposant une zone de texte, une analyse de contenu a été réalisée. Elle a consisté en un examen systématique et méthodique des réponses formulées aux questions administrées.

Cette même méthodologie a été appliquée pour les entretiens retranscrits. Trois axes ont été identifiés en amont par le travail de recherche : i) définition de l'objet d'étude, l'écrit professionnel en fin de formation des enseignants débutants ; ii) l'impact du directeur de mémoire sur ce travail d'écriture et sur sa soutenance face à un jury ; iii) l'éventuel rôle que peut jouer ce travail d'écriture dans l'entrée dans le métier et dans la professionnalisation de ces débutants. D'autres auraient pu être explorés tels que la temporalité de ce travail d'écriture pour l'EFS ou le rôle de la soutenance dans la professionnalisation et auraient eu toute leur pertinence dans cet article. Mais la multiplication des données et de leur analyse induit de la confusion dans l'écriture et l'analyse des résultats. Nous avons volontairement limité notre exploration aux trois axes évoqués ci-dessus et dont le détail suivra.

\section{Trois axes d'exploration}

Des deux questionnaires et des entretiens réalisés avec les formateurs, les résultats selon les trois axes prédéfinis ont été extraits. Ils sont synthétisés dans le texte ci-après.

\subsection{Axe 1 - définition des écrits}

À la lecture des réponses, $42 \%$ ont donné une définition claire et correcte de ces objets. Ils ont évoqué que ce travail consistait à réfléchir sur les pratiques professionnelles qu'ils étaient en train de construire durant cette année : « une réflexion poussée sur l'acte d'enseignement, qui veut nous forcer à sortir d'une pratique naïve pour mieux analyser notre manière de faire » $(\mathrm{H}, 24$ ans, M2). Cependant, un cinquième des EFS ont explicité leur difficulté voire leur incompréhension sur ces travaux. Par des termes comme « une futilité » $\left(\mathrm{F}, 24\right.$ ans, M2) ${ }^{7}$ ou « une apologie du délire didactique » (H, 23 ans, M2), quatre étudiants ont exprimé leur désaccord avec ce travail. Enfin, avec $37 \%$ de non-réponse à cette question, ce résultat mérite un approfondissement. Soit la question "Selon vous, qu'est-ce que l'objet "mémoire" ou "travail réflexif" (suivant celui que vous aviez à 
réaliser) ? » nécessitait une réflexion ou du temps que les répondants n'étaient pas prêts à fournir, soit ils n'avaient pas de réponse à formuler. Cette dernière alternative est plus préoccupante. En effet, il est difficilement concevable de réaliser un travail de niveau Bac + 5 sans être capable de le définir donc d'en comprendre les attendus et la finalité.

Pour les formateurs, les réponses à cette demande de définition ont présenté des difficultés similaires. Seuls onze d'entre eux (8 femmes et 3 hommes) ont complété cet espace libre. Il faudrait peut-être s'interroger sur le fait que des formateurs qui accompagnent des étudiants dans ce type de travail universitaire n'ont pas souhaité ou voulu répondre en définissant une de leurs activités. Autre interrogation, le fait qu'un formateur (H, 46-50 ans, MCF) ${ }^{8}$ a uniquement écrit : « un travail nécessaire ». Voulait-il indiquer l'obligation en fin d'étude universitaire d'avoir à produire ce type d'écrit? Pensait-il davantage à la finalité professionnelle du document ? La réponse ainsi formulée questionne plus qu'elle n'informe. Pour les autres propositions de définition, les idées de travail de réflexion, démarche scientifique, analyse de pratiques d'enseignement, ont été les principaux points développés.

41 Durant les entretiens avec les formateurs, nous avons réinterrogé sur ce point. F3 n'a pas donné de définition, mais a repris les contenus attendus de ce type d'écrit. Après que nous avons souligné cette incompréhension, elle a proposé une définition et a suggéré que «tous [EFS] fassent un mémoire». F4, qui a débuté depuis peu dans l'accompagnement des mémoires, a répondu que la finalité de ces objets lui échappait encore un peu et a conclu par « ta question m'embête».

La définition de ce travail écrit semble peu précise pour près de la moitié des étudiants. Ce point a été éludé tant par les formateurs $(35 \%)$ que par les étudiants. Ces résultats tendraient à montrer que non seulement les étudiants ne perçoivent pas nettement les attendus et finalités de ce travail d'écriture, mais que, pour les formateurs, il revêt également une question sensible. Il semble en effet difficile de mobiliser des étudiants sur un travail quand, en tant que formateur pour celui-ci, la personne n'est pas apte à le définir. Il est toutefois envisageable que, face au questionnaire électronique, les mots pour définir cet écrit aient fait défaut alors que, face aux étudiants, ce même formateur trouvera les termes pour expliquer le travail demandé.

\subsection{Axe 2 - rôle du directeur de mémoire dans l'écrit et la soutenance}

Sur cet axe, dans les réponses des EFS, quatre tendances se sont dessinées. Tout d'abord, ceux (41\%) qui ont considéré le rôle du directeur comme étant une aide pour réaliser le travail d'écriture: «il a lu le travail afin de m'aider dans mes démarches et les modifications à apporter. Il a aussi été présent pour me proposer des sources, des articles scientifiques » (F, 32 ans, M2). Ce qui a été résumé en trois verbes par un étudiant: " accompagner, guider, réorienter " ( $\mathrm{H}, 42$ ans, $\mathrm{M} 2)$. À côté de ces commentaires plutôt positifs, d'autres EFS ( $25 \%$ ) ont considéré que le directeur de mémoire n'avait aucun rôle, voire qu'ils n'avaient « pas de directeur » (H, 39 ans, DUE), ce qui peut interroger. Parmi ceux qui ont souligné l'absence de rôle, trois ont précisé être responsables de cette absence d'échanges, ayant commencé le travail demandé tardivement. Le lien entre la direction du mémoire et la soutenance a conduit cinq EFS à s'interroger sur le rôle que pouvait avoir un directeur de mémoire qui est également membre du jury lors de la soutenance: «il devrait avoir un rôle de guide et nous faire part de ses impressions 
concernant notre travail. Le problème, c'est qu'étant évaluateur de ce travail, il perd ce rôle » (F, 23 ans, M2). Enfin, des points négatifs ont été exprimés sur ce rôle par les mêmes étudiants qui avaient apporté ce type de commentaires sur les écrits.

Concernant la préparation à la soutenance de leur mémoire, 74,5\% des répondantsétudiants ont précisé ne pas avoir sollicité de conseil à leur directeur de mémoire. Pourtant, 40,4\% ont indiqué avoir reçu des conseils de sa part pour l'oral, le directeur de mémoire ayant été présent dans $80 \%$ des soutenances. Cette partie du questionnaire ne concernait théoriquement ${ }^{9}$ pas les EFS inscrits en DU, la soutenance ne faisant pas partie des attendus de leur diplôme à l'ESPE Clermont-Auvergne. Dans leurs réponses, des sentiments de soulagement: « je n'ai pas fait de soutenance pour le travail réflexif et je trouve cela très bien !» (F, 28 ans, DUE) ont côtoyé de la déception et des interrogations : «nous n'avons aucun retour sur notre travail, à part la note, ce qui est décevant » (F, 23 ans, DUE) ; « le travail réflexif, je me demande à quoi il sert : à se poser des questions tout seul dans son coin?» (F, 35 ans, DUE).

Lors des entretiens, les formateurs avaient exprimé le même ressenti sur la nonsoutenance des EFS inscrits en DU. F4 a évoqué son amusement d'entendre ses étudiants être « frustrés de devoir se contenter de si peu ». Les propos rapportés par F7 ont été plus critiques: "les étudiants jugent un peu cavalier qu'il n'y ait pas de soutenance. Il est difficile pour eux de n'avoir comme évaluateur qu'une personne ». Des réponses récoltées dans le cadre du questionnaire sont ressorties deux attentes de la soutenance: les étudiants doivent «témoigner d'une capacité à rendre compte de pratiques d'enseignement/apprentissage en s'appuyant sur des outils scientifiques » (F, 51-55 ans, $\mathrm{MCF}$ ), mais également «témoigner de leur expertise dans le domaine choisi et parler de leur vision du métier presque entre pairs » (F, 46-50 ans, PRCE). Interrogés sur la manière dont les EFS vivent la soutenance, les retours ont été en demi-teinte entre ceux qui ont pensé que ce moment était plutôt bien vécu et ceux qui ont considéré que les étudiants le vivaient comme « un passage obligé » $(\mathrm{F},+60$ ans, PRCE), « la liberté [étant] au bout du tunnel... vite se débarrasser ! 》 (H, 36-40 ans, PRCE).

Concernant le travail écrit, cinq des formateurs ayant participé à l'entretien individuel ont inventorié trois catégories d'EFS : 1) ceux qui se sont investis dans le travail, qui ont su s'en emparer et le faire évoluer ; 2) ceux, qualifiés de « ventre mou » (F5), qui ont fait le travail, mais pour qui la démarche a été laborieuse, émaillée de difficultés méthodologiques, rédactionnelles... ; 3) ceux qui ont fait et rendu un travail a minima, souvent écrit en totalité les jours précédant la date limite. Pour F5, chacune de ces catégories correspond à peu près à un tiers des effectifs. Deux formateurs ont noté une évolution des étudiants dans leur investissement pour ce travail. F2 a précisé que, lorsqu'il est arrivé à l'ESPE, il y a cinq ans, « c'était très difficile. Et plus les années passent et plus j'ai le sentiment que ça s'étouffe un peu ce sentiment de rébellion ». Selon lui, cette évolution serait liée aux actions qu'il a conduites : «j'essaie vraiment de le vendre [le mémoire]. Je leur dis bien qu'après, pris dans la matrice de l'Éducation nationale, ils risquent de regretter ces temps de réflexion ». Pour F3, l'évolution notée proviendrait de la formation en amont du M2: «beaucoup de nos étudiants proviennent de notre M1, donc l'initiation à la recherche de M1 apporte. C'est spectaculaire ».

47 Les principaux résultats de l'axe 2, basés sur des aspects relationnels et humains entre l'EFS et son directeur de mémoire, soulignent des tensions et des divergences sur la manière d'appréhender leurs échanges. L'aide des formateurs a été jugée précieuse par 41 $\%$ des EFS, mais un quart a évoqué l'absence de relations, reconnaissant pour certains que 
la faute leur incriminait : pas de demande ou travail fait trop tardivement pour permettre des échanges ou des relectures. Enfin, une minorité (7\%) a manifesté une forme d'agressivité pour qualifier cette relation. Pour les EFS inscrits en DU, la soutenance n'était pas la finalité du travail d'écriture. Un tiers a vécu ce point avec soulagement, considérant que l'aspect réflexif se suffisait par lui-même pour les aider dans leur professionnalisation. Le second tiers a regretté l'absence de soutenance et plus particulièrement le non-retour sur la production écrite. Le dernier tiers a posé l'aspect factuel de la non-soutenance sans la commenter.

L'insatisfaction des étudiants de master dans la relation pédagogique qu'ils entretiennent avec leur directeur de mémoire a donné lieu à de nombreuses études. Dans sa thèse, Gérard (2009) recense une dizaine de travaux corroborant les siens : entre 30 et $60 \%$ des étudiants se déclarent insatisfaits de leur directeur de mémoire en master. Le moindre résultat obtenu dans notre enquête laisserait supposer que, malgré des insatisfactions exprimées, les EFS apprécieraient l'encadrement de ce travail d'écriture.

Cette insatisfaction est à double sens: les formateurs éprouvent également des sentiments partagés sur l'implication des EFS dans ce travail d'écriture. Les retours qu'ils ont faits ont permis de faire ressortir trois types d'étudiants: ceux qui s'impliquent rapidement dans le travail, ceux qui font le travail au moins-disant, ceux qui éprouvent des difficultés, mais qui cherchent à progresser. Parmi ces derniers, certains, en reconversion professionnelle, éprouveraient non seulement des difficultés pour réaliser ce travail d'écriture, mais également pour se professionnaliser.

\subsection{Axe 3 - impact des écrits dans la professionnalisation}

Deux questions interrogeaient les EFS sur l'impact du travail qu'ils avaient réalisé sur leur professionnalisation : une première pour l'année scolaire à venir et une seconde avec un horizon temporel plus lointain («dans votre métier»). Nous détaillons les réponses obtenues.

51 Pour l'année à venir, les résultats ont montré un équilibre dans les avis. 51,9\% des EFS ont pensé que ce travail écrit leur servirait d'ici moins d'un an. Ainsi, trois domaines ont été recensés :

- pour réaliser leur activité professionnelle: "toute la recherche me sera utile dans la préparation des cours et la mise en place d'activités d'apprentissage pour mes élèves » (F, 39 ans, DUE) ;

- pour analyser leur propre activité: «je pourrai repérer les différentes situations plus facilement et agir avec les bonnes attitudes » (F, 24 ans, M2) ;

- pour poursuivre le travail amorcé : «cela m'a donné envie de poursuivre mes recherches » (F, 25 ans, M2).

Lorsque la temporalité est plus longue (impact au niveau du métier et au-delà de l'année suivante), les réponses positives ont progressé de 6 points : 57,7\% des répondants ont jugé que ce travail leur servirait. La proximité des deux questions a conduit six répondants à renvoyer à la réponse précédente, éludant par là même la partie commentaire. Pour les autres, l'impact envisagé s'est situé majoritairement (66,66 \%) à un niveau de veille scientifique : « envie de se sentir informé sur l'avancée des recherches en sciences de l'éducation pour adapter mes pratiques et prendre en compte l'épanouissement des élèves » (F, 28 ans, DUE). 
53 De ces deux questions, il est donc ressorti que les EFS n'étaient qu'à une faible majorité d'accord sur le fait que le travail réalisé allait leur servir durant l'année ou les années à venir. Comment les formateurs ont-ils perçu cet impact?

Pour aborder ce point, les questions portaient sur les rencontres que les formateurs avaient pu avoir avec d'anciens étudiants. Sept ont répondu positivement. Des commentaires sont ressorties deux tendances opposées, d'égale importance. Soit les retours obtenus par les formateurs ont été positifs (les propos entre guillemets sont ceux des ex-EFS rapportés par les formateurs et entre parenthèses, l'identification du formateur source) : «ce qu'on a fait avec vous, ça nous sert. On repense à ce que vous nous avez montré » (F5) ; soit les échanges ont été empreints d'amertume : «il n'est pas possible de se consacrer à un vrai travail de recherche sur un temps aussi court » ( $F$, 36-40 ans, PRAG). Ces éléments très mitigés sont parfaitement résumés par la réponse de cette formatrice : "très variable suivant les étudiants » (F5), ce qui peut s'appliquer à beaucoup de réponses obtenues.

55 L'impact professionnel immédiatement perçu par les formateurs a également été interrogé. Pour cette question, seule une personne a répondu positivement. Elle a noté : « des progrès parfois dans les aspects didactiques lors des secondes visites de classe, une volonté de rechercher une bibliographie sur d'autres thèmes que celui traité en mémoire » (F, 36-40 ans, PCRE). Les retombées positives sur la professionnalisation resteraient donc soit invisibles aux yeux des formateurs, soit inexistantes lors de l'année de stage. Une étude plus approfondie serait donc nécessaire sur ce point.

Enfin, durant les entretiens, deux formateurs ont exprimé des sentiments d'illégitimité en tant que formateurs en méthodologie de recherche comme en accompagnement de travaux. F2 a inclus dans son ressenti une des collègues de sa discipline avec laquelle il a échangé à ce sujet : « on est assez mal à l'aise. On n'a pas forcément fait de la recherche. Parfois, on a l'impression d'être un peu des imposteurs ». F3 nous a précisé : « la première année où j'étais directeur de mémoires, ça a vraiment été des moments très difficiles pour moi, à savoir est-ce que ma place était bien là ».

57 L'impact de ces écrits dans la professionnalisation des EFS ne trouve pas de réel consensus. Une faible majorité des étudiants a considéré que cet écrit apporterait à leur métier, mais dans une temporalité qui se situerait plutôt à moyen terme que pour l'année qui suit leur titularisation. Bousculés par une année où ils ont eu, en moyenne, neuf heures de cours à préparer, ils sont conscients que l'année suivante, avec un doublement d'heures à assurer devant élèves et avec de nouveaux niveaux d'enseignement, sera encore difficile. L'apport en matière de professionnalité qu'apporteraient ces écrits nécessiterait un accompagnement plus important que celui que nous avons identifié précédemment. Des travaux ont déjà été conduits sur l'inefficience à laisser les débutants découvrir seuls leur activité professionnelle (Ria, Leblanc, Serres \& Durand, 2006).

Les formateurs semblent plus confiants sur les retombées positives des écrits. Les retours qu'ils ont pu avoir avec d'anciens étudiants les ont confortés sur la pertinence de ces écrits dans la professionnalisation des enseignants débutants. Cependant, le caractère scientifique peut être questionné. Selon les pistes de définition élaborées, il apparaîtrait que seuls des enseignants chercheurs devraient assurer l'encadrement de ces travaux. Or, ce statut ne représente en moyenne que 30 \% des enseignants des ESPE (Ginestié, 2017), ce qui serait insuffisant pour le nombre d'EFS à encadrer. De même, les travaux tels qu'évoqués par les répondants, EFS et formateurs, correspondent peu à des écrits 
scientifiques. Il conviendrait donc de revoir le terme et de définir plus précisément les attendus, tant au niveau de la forme que du fond.

\section{Mémoire et travail scientifique réflexif : des écrits sous tension}

différemment selon leur histoire, leur parcours universitaire et/ou professionnel, en négatif comme en positif. D'ailleurs, les critiques recueillies actuellement (Francfort, Joux \& Sebbah, 2017) trouvent écho dans celles qui ont été collectées précédemment (Charles \& Clément, 1997 ; Crinon \& Guigue, 2006 ; Rayou \& van Zanten, 2004), lorsque la formation des enseignants stagiaires se déroulait dans les Instituts universitaires de formation des maîtres (IUFM). Des propos échangés avec les formateurs ou de ceux confiés par les EFS comme les formateurs dans les questionnaires, dans les trois axes présentés et synthétisés, nous avons identifié de grandes tendances sur les causes de ces dichotomies perçues. Ainsi, quatre tensions sont recensées qui pourraient expliquer l'origine des dissentiments des étudiants face à ces travaux.

\subsection{Tensions professionnelles sous contraintes temporelles}

Les EFS sont dans une année où ils doivent tout à la fois assurer des cours devant des élèves, leur objectif professionnel, et continuer de se former pour obtenir le titre nécessaire pour leur titularisation $(\mathrm{bac}+5)$. Le temps qu'ils consacrent aux activités autres que pédagogiques est assimilé à une perte de temps. Ainsi, elles deviennent pour eux une contrainte qui amène leur activité à être empêchée. Clot (2015) l'explique : «la souffrance trouve son origine dans les activités empêchées, qui ne cessent pourtant pas d'agir entre les travailleurs et en chacun d'eux sous prétexte qu'elles sont réduites au silence dans l'organisation » (p. 165). De plus, l'écriture d'un mémoire ne correspond pas aux préoccupations inhérentes à leur nouvelle activité professionnelle : faire classe (Ria, 2001). Toutes leurs préoccupations de débutants percutent leurs obligations universitaires dont fait partie le mémoire/TSR, d'où ce phénomène de rejet pour certains d'entre eux. Ils diffèrent ce travail et tardent à le produire. En résultent des écrits réalisés dans les derniers jours précédant la date limite de dépôt, pour lesquels la réflexion attendue n'a pas atteint la maturation souhaitée (Bruillard, 2017). De même, en retard dans la réalisation comme dans les rendus, les EFS esquivent les rencontres avec leur directeur, se privant d'un accompagnement faisant partie intégrante de leur professionnalisation.

\subsection{Tensions identitaires}

61 Nommés administrativement étudiants fonctionnaires stagiaires, certains d'entre eux éprouvent des difficultés à se situer identitairement. En tant qu'étudiants, leur cursus universitaire s'achève par un travail d'écriture de type mémoire ou TSR. En tant que fonctionnaires stagiaires, ils sont en attente d'une titularisation de leur employeur. En tant que jeunes enseignants, ils cherchent à se construire une identité professionnelle. Ces tensions identitaires entre le lieu de stage et la formation universitaire, déjà constatées dans de nombreux travaux (Durand, 2000 ; Dozolme, 2015 ; Guibert, Lazuech \& 
Rimbert, 2008 ; Zimmermann, 2013) se trouvent exacerbées par la réalisation du travail d'écriture. Pourtant, Zimmermann (2013) note l'impact positif qu'aurait la tenue d'un carnet de bord pour la formation des stagiaires, leur permettant une mise à distance et une analyse réflexive, facilitant ainsi leur construction identitaire. Le mémoire ou le travail scientifique réflexif ont ces mêmes objectifs. Toutefois, si, sur le terrain, le résultat du travail expérimental mené s'avère être un échec, comment, en tant que chercheurs et professionnels débutants, vont-ils traduire cela dans leurs écrits? La lecture de ceux-ci est actée par les formateurs, qui sont aussi évaluateurs, par le tuteur terrain, et par, éventuellement, le corps d'inspection (Crinon \& Guigue, 2006). De même, lors de la soutenance, leur prestation montre aussi d'éventuelles insuffisances. Donc, les EFS se trouvent partagés entre une expérimentation hasardeuse, source d'une image dommageable, et ce, à quelques jours de leur titularisation, et un travail a minima, mais sécurisant. Cette dualité des positions entre chercheur et formé est notée par Reuter (2004, p. 23), parlant d'une « oscillation entre une position trop basse et une position trop haute ».

\subsection{Tensions relationnelles}

62 Pendant cette année de formation se construit une relation étudiant-directeur de mémoire. En licence, un étudiant entretient une relation groupale avec l'enseignant, dans le cadre de cours communs comportant les mêmes apprentissages. En année de stagiairisation, la réalisation du mémoire/TSR déstructure cette organisation : la relation devient duale et la dimension institutionnelle conduit à une autodirection de l'apprenant. Ces modifications de fonctionnement nécessitent que l'étudiant revoie son rapport à la formation et accepte de l'envisager différemment. De plus, le type de relation étudiantdirecteur devrait relever de la dyade, chacun apportant à l'autre, loin du mandarinat qui existait autrefois. Cependant, ce tandem où chacun a des attendus est malmené par des incompréhensions, notamment autour des « dialogues critiques constructifs » (Gremmo \& Gérard, 2008). Dans ce contexte, le directeur de mémoire souhaite accompagner l'étudiant dans un double objectif : l'aider à se positionner en tant qu'apprenti-chercheur et le faire progresser dans sa recherche. Or, beaucoup d'étudiants vivraient mal ce temps d'échange, «non pas d'un point de vue scientifique et développemental, mais comme un rejet de toute leur personne»(p.52). Par leurs réponses, les EFS enquêtés éprouveraient également des difficultés à faire cette distinction, ayant des termes rudes à l'égard de leur directeur de mémoire. Cette relation est également mise à mal par les modalités d'évaluation du travail d'écriture. Jugés par leur directeur sur le travail écrit rendu comme lors de la soutenance, les EFS doutent de son impartialité, trouvant difficile d'être à la fois conseiller et évaluateur. Cette modalité de contrôle pourrait donc être aussi un élément minorant l'adhésion à ce travail.

\subsection{Tensions rédactionnelles}

Écrire consiste à transformer ce qui peut se résumer à un amas de notes, de lectures, d'expérimentations en un projet qui va exister, sera communiqué et, comme nous l'avons évoqué, appelé à être jugé. De nombreux travaux (Altet, Bru \& Blanchard-Laville, 2012 ; Bouissou \& Aroq, 2005 ; Crinon \& Guigue, 2006 ; Mead, 2006...) montrent l'impact positif de l'écriture dans le développement de la pensée et de la réflexion sur les pratiques comme pour sa propre pratique. Il s'agit donc d'un exercice qui « renvoie à un ensemble 
de pratiques et de genres peu facile» (Reuter, 2004, p. 25), d'autant plus quand l'écriture se veut scientifique. Il peut devenir source de difficulté pour nombre d'étudiants. La diversité des cursus universitaires, des parcours professionnels pour les EFS en reconversion explique en partie les difficultés éprouvées. Ces mêmes paramètres empêchent également toute généralisation des données. Il conviendrait donc de ne pas survaloriser l'écrit et les procédures ad hoc, au détriment de tout le processus de réflexion et d'élaboration d'expérimentation qui est mis en œuvre en amont, réel acteur de la professionnalisation (Bouissou \& Aroq, 2005).

Ces différentes tensions répertoriées fournissent un début d'explication sur les causes du rejet chez certains EFS de cet écrit de fin de cursus universitaire. Pris par les préoccupations inhérentes à l'acquisition d'un nouveau métier, ils éprouvent des difficultés à considérer la pertinence de ce travail dans leur professionnalisation. Pensé et écrit souvent trop tardivement, il est élaboré sans réelle implication, correspondant peu ou pas à une démarche réflexive. Si l'institution étaye sur son argumentation sur cet objectif de professionnalité (note du 28/10/2015), nous assistons à une remise en cause du volet réflexif comme étant le seul porteur de professionnalité (Gomez, 2001). Ainsi le modèle de la pratique réflexive de Schön (1994) présenterait le risque d'une réflexion autonome et individuelle, uniquement dirigée vers un changement immédiat. Or, tout le travail réalisé autour de ces écrits demande une ouverture sur son environnement professionnel, des temps d'échange avec les différents acteurs, avec son directeur de mémoire. De même, l'objectif de ces travaux s'inscrit dans une dynamique qui ne saurait se limiter à hic et nunc. Même si l'impact positif de ces écrits parait incontestable, il y aurait quand même un danger à considérer que ce travail d'écriture résoudrait tous les problèmes des enseignants débutants et leur garantirait un cheminement professionnel serein.

\section{Pour conclure}

Ce travail d'écriture de fin de cursus universitaire devrait conduire les EFS à une activité réflexive sur leurs pratiques professionnelles débutantes, à un accomplissement de soi et à une satisfaction finale au terme de la production de ces documents. Cependant, de notre travail ressortent plusieurs points montrant les faiblesses de l'impact de ces écrits sur la professionnalisation face aux attendus institutionnels forts. Tout d'abord, la définition même de ces travaux ne semble pas circonscrite, que ce soit de la part des EFS comme de certains formateurs. Cette variabilité provient à la fois des parcours des répondants comme de leur familiarité ou non à cet exercice. De même, les attentes en matière d'encadrement divergent. Si certains étudiants ont profité du questionnaire pour donner libre cours à leur ressenti, d'autres ont précisé que l'absence d'encadrement était de leur ressort. Un travail d'écriture réalisé quelques jours avant la date limite de rendu n'a laissé aucune place à des temps de rencontres et d'échanges, moments propices pour la réflexivité sur leur activité. De leur côté, les formateurs, en attente de ces temps, ont estimé que les EFS doivent en être à l'origine. Pourtant, ces moments permettraient aux EFS de revenir sur leur activité et de s'interroger, tout en questionnant l'encadrant, sur leur pratique, à l'aune des lectures scientifiques réalisées (Bouissou \& Aroq, 2005). Ainsi, tout l'impact que pourraient avoir ces écrits sur la professionnalisation prendrait forme (Morisse et al., 2012). Or, cette professionnalisation par l'écrit se dessine modestement dans les réponses des EFS, la moitié d'entre eux seulement envisageant un impact positif 
et plutôt à moyenne échéance. Durant leur année de stage, les préoccupations de ces enseignants débutants sont focalisées sur leur activité en classe, auprès des élèves. La production d'un écrit dont ils ne perçoivent pas la finalité dans leur activité (Wittorski, 2008b) entre en conflit avec ces préoccupations, rendant ainsi la finalité inopérante.

66 Avec ce travail autour des écrits réflexifs dans la formation des enseignants débutants, des arbitrages ont été conduits. D'autres auraient pu être mis en œuvre. Ainsi, la place occupée par le mémoire et le travail réflexif mériterait d'être repensée. Une plus grande valorisation de ces travaux mériterait réflexion. Une diffusion plus large au sein de la sphère éducative pourrait devenir un facteur de motivation pour les EFS (Crinon \& Guigue, 2006) et une source de réflexion pour la communauté. Autre piste qui aurait pu être explorée: les interférences générées par le nombre important de partenaires gravitant autour de ces objets et pouvant entrer en contradiction (Bouissou \& Aroq, 2005 ; Mead, 2006; Rayou \& van Zanten, 2004). Comment de jeunes professionnels en construction identitaire parviennent-ils à faire abstraction d'informations pouvant être contraires autour de ce travail d'écriture, tout en adoptant une posture d'analyse de leur activité ? Une indispensable concertation des acteurs de leur professionnalisation autour des attendus offrirait aux EFS l'opportunité de se construire de façon plus satisfaisante.

\section{Annexes}

\section{Annexe 1 : questionnaire à l'attention des EFS ${ }^{10}$}

\section{Identification}

Quel âge avez-vous?

Êtes-vous? Homme - Femme

Dans quel diplôme êtes-vous inscrit(e) en 2016-2017 ?*

Quel est votre dernier diplôme universitaire obtenu avant l'année universitaire 2016-2017 ?*

Réalisation de mémoire dans le cadre de votre cursus universitaire antérieur

Aviez-vous déjà réalisé un mémoire ou un travail réflexif dans le cadre de votre cursus universitaire antérieur ?*

Si oui, quel avait été votre ressenti sur ce travail?

\section{La formation}

Combien d'heures de formation dédiée à la méthodologie de recherche avez-vous eues ?* Jugez-vous ce nombre d'heures comme suffisant?*

Quelles remarques souhaitez-vous faire?

\section{Le mémoire ou le travail réflexif}

Selon vous, qu'est-ce que l'objet « mémoire » ou « travail réflexif » (suivant celui que vous deviez réaliser)?

Avez-vous eu des difficultés pour... 


\begin{tabular}{|l|l|l|l|l|}
\hline & $\begin{array}{l}\text { Pas du } \\
\text { tout }\end{array}$ & $\begin{array}{l}\text { Un } \\
\text { peu }\end{array}$ & Beaucoup & Énormément \\
\hline Trouver le thème? & & & & \\
\hline Trouver le sujet? & & & & \\
\hline Trouver la bibliographie? & & & & \\
\hline Mettre en place la collecte de données? & & & & \\
\hline $\begin{array}{l}\text { Faire le lien entre cours et document à } \\
\text { produire? }\end{array}$ & & & & \\
\hline
\end{tabular}

À quel moment de l'année universitaire avez-vous écrit votre mémoire/travail réflexif ? Que pensez-vous du moment où s'inscrit ce travail dans votre année d'étudiant(e) fonctionnaire stagiaire?

Des commentaires d'ordre logistique, technique ou réglementaire sur le mémoire/travail réflexif et/ou la collecte de données...

\section{Encadrement du travail}

71 Avez-vous eu des difficultés pour...

\begin{tabular}{|l|l|l|l|l|}
\hline & $\begin{array}{l}\text { Pas du } \\
\text { tout }\end{array}$ & $\begin{array}{l}\text { Un } \\
\text { peu }\end{array}$ & Beaucoup & Énormément \\
\hline $\begin{array}{l}\text { Comprendre la mission d'un directeur de } \\
\text { mémoire? }\end{array}$ & & & & \\
\hline $\begin{array}{l}\text { Trouver les modalités d'échanges avec le directeur } \\
\text { de mémoire? }\end{array}$ & & & & \\
\hline Trouver le temps pour échanger? & & & & \\
\hline
\end{tabular}

Autres commentaires...

\section{Écriture du travail}

Avez-vous eu des difficultés pour...

\begin{tabular}{|l|l|l|l|l|}
\hline & Pas du tout & Un peu & Beaucoup & Énormément \\
\hline Comprendre les attendus de cet écrit? & & & & \\
\hline Rédiger le travail demandé? & & & & \\
\hline Organiser les différentes parties? & & & & \\
\hline
\end{tabular}


Utiliser les citations (forme) ?

Quel a été le rôle du directeur de mémoire dans cette étape du travail ?

Des commentaires sur l'écriture du mémoire...

\section{Soutenance}

Qui était présent à votre soutenance ?*

Avez-vous sollicité des conseils à votre directeur de mémoire pour cette soutenance ?* Avez-vous reçu des conseils de la part de votre directeur de mémoire pour cette soutenance ?*

Comment avez-vous vécu cette épreuve ?*

Si vous n'étiez pas serein(e), pourriez-vous indiquer ce qui, selon vous, a occasionné cet inconfort?

Comment avez-vous considéré l'attitude des membres du jury ? *

Des commentaires sur la soutenance du mémoire...

\section{Et la suite...}

Si vous aviez à refaire votre mémoire/travail réflexif, changeriez-vous quelque chose ?*

Si oui, quel(s) élément(s)?

Pensez-vous que votre mémoire/travail réflexif vous servira pour l'année scolaire à venir ?*

Si oui, de quelle manière?

Pensez-vous que votre mémoire/travail réflexif vous servira plus tard dans votre métier ?*

Comment pensez-vous qu'il impactera votre futur métier?

Pensez-vous que votre mémoire/travail réflexif est susceptible d'intéresser d'autres enseignants ?*

Commentaires

Connaissez-vous le concours « mon mémoire en 180 secondes » ?*

Si oui, avez-vous candidaté ?

Votre ressenti à propos de ce concours :

Suite à ce travail de recherche, envisageriez-vous de faire une thèse en sciences de l'Éducation ?*

Si oui, quel en serait le thème?

\section{Annexe 2 : questionnaire à l'attention des formateurs}

\section{Identification}

Dans quelle tranche d'âge vous situez-vous ?*

Êtes-vous? Homme - Femme

Quel est votre statut au sein de l'ESPE Clermont-Auvergne ?*

Depuis combien d'années pratiquez-vous cette activité de cours/accompagnement à l'ESPE ?*

Exercez-vous des fonctions similaires (cours de méthodologie/encadrement de mémoire) dans un autre diplôme ou une autre formation de l'ESPE ?* 
Si oui, lequel/laquelle?

Exercez-vous des fonctions similaires (cours de méthodologie/encadrement de mémoire) dans une autre composante de l'UCA ?*

Si oui, laquelle?

N’encadrez-vous que des mémoires, sans faire de cours de méthodologie ? *

\section{Cours de méthodologie de recherche}

Combien d'heures de formation dédiées à la méthodologie avez-vous réalisées ? *

Jugez-vous ce nombre d'heures comme suffisant ?*

Commentaires

\section{Accompagnement mémoire}

Pensez-vous que les étudiants ont éprouvé des difficultés pour :

\begin{tabular}{|c|c|c|c|c|}
\hline & $\begin{array}{ll}\text { Pas } & d u \\
\text { tout }\end{array}$ & $\begin{array}{l}\text { Un } \\
\text { peu }\end{array}$ & Beaucoup & Énormément \\
\hline Trouver le thème? & & & & \\
\hline Trouver le sujet? & & & & \\
\hline Trouver la bibliographie? & & & & \\
\hline Mettre en place la collecte de données? & & & & \\
\hline Faire le lien entre cours et document à produire? & & & & \\
\hline Comprendre les attendus de cet écrit? & & & & \\
\hline Rédiger le travail demandé? & & & & \\
\hline Organiser les différentes parties? & & & & \\
\hline Utiliser les citations (forme)? & & & & \\
\hline $\begin{array}{l}\text { Trouver le positionnement attendu lors de la } \\
\text { soutenance? }\end{array}$ & & & & \\
\hline
\end{tabular}

Commentaires

Professionnalisation des étudiants par le mémoire/travail réflexif

Comment définiriez-vous l'objet « mémoire » ou « travail réflexif »?

Selon vous, cela aurait-il du sens de considérer le mémoire/travail réflexif comme un objet « en soi », indépendamment de l'ensemble de la formation (master MEEF/DU) dans lequel il prend place ?*

Commentaires 
Selon vous, le mémoire/travail réflexif existerait-il plutôt en lien/en relation avec d'autres expériences ? ${ }^{*}$

Commentaires

\section{Temporalité et impact du mémoire}

Selon vous, pour les étudiants fonctionnaires stagiaires (EFS) :

\begin{tabular}{|l|l|l|}
\hline & Oui & Non \\
\hline Le mémoire/travail réflexif est écrit « à la fin » d'une réflexion. & & \\
\hline Le mémoire/travail réflexif est un moyen de cette réflexion. & & \\
\hline Le mémoire/travail réflexif est un outil formatif au moment de sa production. & & \\
\hline Le mémoire/travail réflexif s'inscrit dans une continuité de formation. & & \\
\hline $\begin{array}{l}\text { Le mémoire/travail réflexif s'avérera être plus formatif dans la suite de leur } \\
\text { professionnalisation. }\end{array}$ & & \\
\hline
\end{tabular}

Commentaires

\section{Soutenance mémoire}

Selon vous, quel rôle peut jouer la soutenance dans la professionnalisation des EFS ?

Comment pensez-vous que les EFS vivent cet oral?

\section{Retour sur les anciennes promotions}

81 Avez-vous eu des contacts avec d'anciens EFS à propos du mémoire ? *

Pourriez-vous résumer vos échanges?

Avez-vous pu évaluer l'impact professionnel de ce travail dans la professionnalisation des EFS précédemment formés ? *

De quelle manière?

Que vous ayez eu des retours de vos anciens étudiants ou non, pensez-vous avoir fait évoluer votre manière d'encadrer les mémoires ?*

Comment?

\section{BIBLIOGRAPHIE}

Altet, M., Bru, M. \& Blanchard-Laville, C. (2012). Observer les pratiques enseignantes. Paris :

L'Harmattan. 
Barbier, J.-M. (1996). De l'usage de la notion d'identité en recherche, notamment dans le domaine de la formation, Éducation permanente, 128, 11-26.

Beauvoir, J.-L. \& Deschamps, J.-C. (1990). Vers la cognition sociale. Dans C. Bonnet, R. Ghiglione et J.-F. Richard (dir.), Traité de psychologie cognitive. 3, cognition, représentation, communication (pp. 3-110). Paris : Dunod.

Berthelot, J.-M. (2003). Figures du texte scientifique. Paris : PUF.

Bouissou, C. \& Aroq, C. (2005). Mémoires professionnels et développement des enseignants en formation. Étude des rapports aux savoirs et des rapports à l'action. Revue des sciences de l'éducation, 31, 15-31.

Bouquet, B. (2009). Diversité et enjeux des écrits professionnels. Vie sociale, 2, 81-93.

Bourdoncle, R. (2000). Professionnalisation, formes et dispositifs. Recherche et formation, 35, 117-132.

Bruillard, É. (2017). Former des enseignants pour une école « numérique » et « postmoderne ». Administration \& Éducation, 154, 25-31.

Campion, M.-D. (2017). Mise en place du projet ESPE et territoire académique. Administration \& Éducation, 154, 117-122.

Charles, F. \& Clément, J.-P. (1997). Comment devient-on enseignant ? L'IUFM et ses publics. Strasbourg : Presses universitaires de Strasbourg.

Chartier A.-M. (2003). Écrire les pratiques professionnelles : réticences et résistances des praticiens. Dans C. Blanchard-Laville et D. Fablet (dir.), Écrire les pratiques professionnelles. Dispositifs d'analyse de pratiques et écriture (pp. 17-56), Paris : L'Harmattan.

Clot, Y. (2015). Le travail à cœur, Paris : La Découverte.

Crinon, J. \& Guigue, M. (2006). Écriture et professionnalisation. Revue française de pédagogie, 156, 117-169.

Dabène, M. (1993). L'adulte et l'écriture. Bruxelles : De Boeck.

Durand, M. (2000). Développement personnel et accès à une culture professionnelle en formation initiale des professeurs. Communication au colloque de l'ACFAS, Montréal.

Dozolme, S. (2015). Les enseignants débutants du second degré issus de l'entreprise (Thèse de doctorat non publiée). Université Blaise Pascal, France.

Dubar, C., Tripier, P. \& Boussard, V. (2011). Sociologie des professions $-3^{\mathrm{e}}$ édition. Paris : Armand Colin.

Filâtre, D. (2015). Recommandations pour le mémoire « MEEF » dans le cadre de l'année de formation des enseignants. Repéré à http://www.snesup.fr/sites/default/files/fichier/rapport_du_cnsfde_memoire-vf_provdf.pdf.

Filâtre, D. (2017). Un bilan des trois premières années d'exercice des ESPE. À partir des travaux du comité national de suivi. Administration \& Éducation, 154, 57-65.

Francfort, D., Joux, A. \& Sebbah, B. (2017). La parole aux étudiants : regards croisés sur la formation en ESPE. Une synthèse d'entretiens avec des étudiants inscrits à Aix-Marseille université. Administration \& Éducation, 154, 93-98.

Gérard, L. (2009). L'accompagnement en contexte de formation universitaire : étude de la direction de mémoire comme facteur de réussite en master (Thèse de doctorat non publiée). Université Nancy 2 , France. 
Ginestié, J. (2017). Former à l'université des professionnels de l'enseignement, de l'éducation et de la formation. Administration \& Éducation, 154, 77-83.

Glorieux, C. (2016). Mémoires professionnels, mémoires d'application et autres travaux de fin d'études (TFE). Namur : Presses universitaires de Namur.

Gomez, F. (2001). Le mémoire professionnel : professionnel ? un qualificatif à interroger. Recherche et formation, 36, 173-189.

Gremmo, M.-J. \& Gérard, L. (2008). Accompagner les apprentis-chercheurs. Jeux et enjeux de la direction de mémoire. Recherche et formation, 59, 43-48.

Guibert, P., Lazuech, G. \& Rimbert, F. (2008). Enseignants débutants : « faire ses classes ». Rennes : Presses universitaires de Rennes.

Hughes, E. C. (1996). Le regard sociologique. Paris : Éditions de l'école des hautes études en sciences sociales.

Lahire, B. (1998). L'homme pluriel. Les ressorts de l'action. Paris : Nathan.

Lahire, B. (2001). De la théorie de l'habitus à une sociologie psychologique. Dans B. Lahire (dir.), Le travail sociologique de Pierre Bourdieu (pp. 121-152). Paris : La Découverte.

Lang, V. (1999). La professionnalisation des enseignants. Paris : PUF.

Lefresne, F. \& Fournier, Y. (2016). L'Europe de l'Éducation en chiffres. Paris : MENESR.

Mead, G.-H. (2006). L'esprit, le soi et la société. Paris : PUF.

Morisse, M., Lafortune, L. \& Cros, F. (2012). Se professionnaliser par l'écriture: quels accompagnements? Québec : Presses de l'université du Québec.

Mroue, M. (2014). Écrit de recherche universitaire : éléments pour une sensibilisation au positionnement scientifique à travers la phraséologie transdiciplinaire (Thèse de doctorat non publiée). Université de Grenoble, France.

Rayou, P. \& van Zanten, A. (2004). Enquête sur les nouveaux enseignants. Changeront-ils l'école ? Paris : Bayard.

Reuter, Y. (2004). Analyser les problèmes de l'écriture de recherche en formation. Pratiques, 121/122, 9-27.

Ria, L. (2001). Les préoccupations des enseignants débutants en éducation physique et sportive - Étude de l'expérience professionnelle et conception d'aides à la formation (Thèse de doctorat non publiée). Université de Montpellier I, France.

Ria, L., Leblanc, S., Serres, G. \& Durand, M. (2006). Recherche et formation en « analyse de pratiques ", un exemple d'articulation. Recherche et formation, 51, 43-56

Schön, D. A. (1994). Le praticien réflexif. À la recherche du savoir caché dans l'agir professionnel. Montréal : Éditions Logiques.

Vermersch, P. (1996). L'entretien d'explicitation. Paris : ESF Éditeur.

Wittorski, R. (2008a). La professionnalisation. Savoirs, 17, 9-36.

Wittorski, R. \& Briquet-Duhazé, S. (2008b). Introduction. Dans R. Wittorski \& S. Briquet-Duhazé (dir.) Comment les enseignants apprennent-ils leur métier ? (pp. 11-32). Paris : L’Harmattan.

Zimmermann, P. (2013). Analyse du façonnage de l'identité professionnelle des enseignants d'école primaire en formation initiale (Thèse de doctorat non publiée). Université de Nice - Sophia Antipolis, France. 


\section{NOTES}

1. Évocation dans le rapport de 2015 d'un rapport datant de l'été 2014 élaboré par le Comité national de suivi et faisant part de recommandations sur le fond et la forme de l'élaboration du mémoire.

2. Ce chiffre correspond à l'ensemble des EFS, premier degré, second degré et conseillers principaux d'éducation.

3. Compétences en linguistique, sémiotique, pragmatique et sociologie.

4. 230 étudiants fonctionnaires stagiaires dans le $2^{\mathrm{d}}$ degré, 17 parcours disciplinaires différents.

5. Diplôme universitaire préparé par les lauréats du concours soit détenteurs d'un master, soit dispensés de titre et/ou ne pouvant entrer directement en master MEEF $2^{\mathrm{e}}$ année.

6. Diplôme universitaire proposé à ceux qui n'ont pas été titularisés lors de leur année de M2, mais qui ont validé leur master (renouvellement de stage).

7. F : Femmes - H : Hommes - M2 : étudiant en M2 MEEF - DUE : DU Enseigner dans le second degré.

8. PRCE : enseignant certifié - PRAG : enseignant agrégé - MCF : maître de conférences - PU : professeur d'université.

9. 5 des 15 étudiants en DU ont complété cette partie du questionnaire bien qu'ils ne soient pas concernés.

10. L'astérisque qui suit les questions indique que le répondant disposait soit d'un choix oui-non soit d'une liste de réponses possibles.

\section{RÉSUMÉS}

L'initiation à la recherche ainsi que la rédaction d'un écrit comptent parmi les exigences attendues dans le cadre de la formation initiale des enseignants en France. Selon leur parcours universitaire et professionnel, il est proposé à ces débutants de réaliser soit un mémoire, pour les étudiants du master Métier de l'enseignement de l'éducation et de la formation (MEEF), soit un travail scientifique réflexif (TSR) pour ceux qui sont inscrits en diplôme universitaire (DU). La réalisation de ces travaux constitue un point névralgique dans la formation au métier d'enseignant, sujet de controverses. Dans le cadre d'une enquête réalisée auprès des étudiants fonctionnaires stagiaires (EFS) et des formateurs de l'ESPE (École supérieure du professorat et de l'éducation) Clermont-Auvergne, trois axes ont été explorés : la définition de ces écrits par les étudiants comme les formateurs, le rôle du directeur de mémoire dans leur production, l'impact ressenti de ces écrits sur la professionnalisation des enseignants débutants. Ils tentent de comprendre les tensions et attentes à l'égard de ces objets.

The initiation to research and the writing of a writing correspond to the requirements expected in the context of initial teacher training in France. Depending on their academic and professional background, it is proposed to these beginners to realize either a dissertation, for the students of the Master of Education and Training, or to carry out a reflexive scientific work for those enrolled in a university degree. The realization of this work is a key point in teacher training, also a subject of controversy. As part of a survey conducted among student young teachers and 
teacher trainers of the ESPE (Higher School of Teaching and Education) Clermont-Auvergne, three axes were explored: the definition of these writings by students and trainers, the role of the directors in their production, the impact of these writings on the professionalization of beginning teachers. We try to understand the tensions and expectations with regard to these objects.

INDEX

Mots-clés : formation initiale des enseignants, mémoire, professionnalisation, travail scientifique réflexif

Keywords : initial teacher training, dissertation, professionalisation, reflective scientific work

\section{AUTEUR}

\section{SYLVIE DOZOLME}

Docteure en sciences de l'éducation, laboratoire Acté (EA 4281), formatrice ESPE ClermontAuvergne 